\title{
Custom-Made Cell Designed for Thermal Studies and In Situ X-Ray Spectroscopy Experiments
}

\author{
K. Wojtaszek, K. Tyrąa And A. WaCH* \\ Institute of Nuclear Physics, Polish Academy of Sciences, PL-31342 Krakow, Poland
}

\begin{abstract}
In situ measurements provide an opportunity for investigating not only the initial and final states of the process, but also intermediate states within the process. In particular, X-ray spectroscopy techniques have become an important tool to obtain relevant information (oxidation state, electronic structure, local bonding environment) during material's transformation under real reaction conditions. Herein, we describe a novel cell specifically designed for ex situ and in situ thermal studies with X-ray spectroscopy techniques. A key feature of this setup is the possibility to work at high temperatures (up to $1370^{\circ} \mathrm{C}$ ) and under different atmospheres (from inert to reactive). The cell is conveniently designed to allow measurements in in-house laboratories as well as large-scale synchrotron facilities. The setup allows also to perform X-ray spectroscopy measurements in both fluorescence and transmission mode. In order to demonstrate the capabilities of the designed cell, thermal oxidation of metallic titanium to titanium dioxide was studied. First, the manufactured cell was tested in the laboratory and ex situ X-ray photoelectron measurements were performed for the Ti metal discs heated at different temperatures. Then, the setup was mounted on SuperXAS beamline at Swiss Light Source, and Ti $K$-edge X-ray absorption spectroscopy and valence-to-core X-ray emission spectra were collected during the thermal oxidation process. The combination of these techniques provided information about the electronic structure of occupied and unoccupied states around the Fermi level.
\end{abstract}

DOI: 10.12693/APhysPolA.137.54

PACS/topics: in situ cell, thermal oxidation, X-ray spectroscopy, titanium

\section{Introduction}

The electronic band structure determines the physical and chemical properties of materials, such as optical absorption, superconductivity, magnetoresistance, catalytic and photocatalytic activity [1-3]. Thus, knowledge about the electronic band structure is of prime importance to understand materials function and reactivity. This information can be obtained by several, conventional spectroscopic techniques, like UV-Vis, the Raman spectroscopy, X-ray and ultraviolet photoelectron spectroscopy (XPS and UPS) [4, 5], which are useful in studying materials under steady-state or in some circumstances in situ conditions. The high demand for better performing materials has pushed the direction of experimental design for in situ measurements, which allow data collection under operando conditions. Hard X-rays (with energy higher than $4 \mathrm{keV}$ ) can penetrate through window materials (e.g., kapton) of in situ cells [6]. This allows determining the structure of materials in their natural or in operando conditions, for example in air, under reactive gases at high temperature and pressure, and in the liquid phase. One of the first in situ cells designed for X-ray absorption spectroscopy studies on catalytic samples was presented by groups of Lytle [7] and Koningsberger [8]. Since then, a number of reaction cells for X-ray spectroscopy measurements, with

*corresponding author; e-mail: anna.wach@ifj.edu.pl different specific design (depending on the system to be investigated and the experimental reaction conditions), have been developed [9, 10]. An general review of the cell-developments may be found in book $X$-ray Absorption Fine Structure for Catalysts and Surfaces, edited by Y. Iwasawa [11]. The combination of X-ray spectroscopy techniques, such as X-ray absorption spectroscopy (XAS) and X-ray emission spectroscopy (XES), with hard X-rays enables to obtain a unique picture of electronic band structure changes under material working conditions $[12,13]$. X-ray absorption spectrum is a reflection of the unoccupied density of states (conduction band), whereas X-ray emission spectrum gives access to the occupied electronic states (valence band), and thus allows for a detailed investigation of the local electronic structure of the absorbing atom.

In the present manuscript, we describe a custom-made cell specifically designed for in situ thermal studies under different atmospheres with X-ray absorption and X-ray emission spectroscopy. The manufactured cell was initially tested in the laboratory, and thermal oxidation of metallic titanium foil to titanium dioxide was used as a probe reaction. Ti metal discs heated at two different temperatures were studied ex situ by means of X-ray photoelectron spectroscopy. Subsequently, to demonstrate the capabilities of the designed cell for in situ measurements, the setup was mounted at the SuperXAS beamline of the Swiss Light Source synchrotron. The non-resonant $\mathrm{X}$-ray emission and X-ray absorption spectra were collected in situ, at the beginning and end of thermal oxidation of metallic titanium. 


\section{Experimental section}

\subsection{Experimental setup}

The titanium metal discs (from Goodfellow, 99.99\%), $10 \mathrm{~mm}$ in diameter and $1.0 \mathrm{~mm}$ thick, were used without further purification. The single disc was mounted in a custom-made cell with a heating element placed below the sample.

\subsection{Ex situ measurements}

For ex situ test measurements, two titanium metal discs were heated under ambient atmosphere from $25^{\circ} \mathrm{C}$ to $300{ }^{\circ} \mathrm{C}$ and $500^{\circ} \mathrm{C}$, respectively. Heating rate program was set at $5^{\circ} \mathrm{C} / \mathrm{min}$ and the samples were maintained at set temperature for $5 \mathrm{~h}$. The samples were labelled as $\mathrm{Ti}$ 300C and $\mathrm{Ti}$ 500C. To evaluate the oxidation process, the XPS measurements were performed. XPS spectra were recorded on a Prevac photoelectron spectrometer equipped with a hemispherical VG SCIENTA R3000 analyzer. The photoelectron spectra were measured using a monochromatized aluminum $\mathrm{Al} K_{\alpha}$ source $(E=1486.6 \mathrm{eV})$. The base pressure in the analysis chamber during the measurements was $5 \times 10^{-9}$ mbar. The binding energies were referenced to C $1 s$ core level $(284.8 \mathrm{eV})$ from hydrocarbon contaminations. The composition and chemical surrounding of the sample surface were investigated on the basis of the areas and binding energies of Ti $2 p, \mathrm{O} 1 s$, and $\mathrm{C} 1 s$ photoelectron peaks. The fitting of high resolution spectra was provided through the CasaXPS software.

\subsection{In situ measurements}

For in situ measurements, two titanium metal discs were heated under ambient atmosphere, at a slow rate $\left(5^{\circ} \mathrm{C} / \mathrm{min}\right)$ from $25^{\circ} \mathrm{C}$ to $700^{\circ} \mathrm{C}$, dwelled at $700^{\circ} \mathrm{C}$ for $30 \mathrm{~min}$ and cooled rapidly to room temperature. The experiment was conducted at the SuperXAS beamline at Swiss Light Source (PSI, Villigen, Switzerland). The X-ray beam from the $2.9 \mathrm{~T}$ superbend magnet was monochromatized by a double $\mathrm{Si}(111)$ crystal monochromator. The photon flux on the sample was $c a .10^{11}$ photons/s. The beamline was calibrated using a $10 \mu \mathrm{m}$ Ti foil (Ti $K$ edge at $4966 \mathrm{eV}$ ). The XAS and non-resonant XES spectra were collected in situ, at the beginning and end of thermal oxidation of metallic titanium. X-ray absorption spectra around the Ti $K$-edge were measured in the fluorescence mode using a five-element sensor silicon drift (SDD) detector. The non-resonant X-ray emission spectra were recorded using a von Hamos type spectrometer at fixed X-ray energy above the ionization threshold (i.e., X-ray photons with $5136 \mathrm{eV}$ energy). The X-rays emitted from the sample were diffracted by a cylindrically bent $\mathrm{Si}(100)$ crystal with a $25 \mathrm{~cm}$ radius of curvature at $67^{\circ}$ and detected on a 2D Pilatus detector.

\section{Results and discussion}

\subsection{Cell design}

The presented cell was designed for in situ thermal studies under different atmospheres with X-ray absorption and X-ray emission spectroscopy. The cell is made of stainless steel and consists of cubic sample chamber with the outer edges length of $70.5 \mathrm{~mm}$ (see Fig. 1). On both, top and bottom side of the chamber, two round apertures are cut in the middle. Through one of the apertures the sample holder/heater is attached to the chamber, the other one is closed. Both sides are sealed by use of copper gasket. The side walls of the sample chamber have additional round windows with diameter of $38 \mathrm{~mm}$. These windows allow for the sample mounting as well as enable both entry of the incident radiation and exit of the emitted fluorescence or transmitted radiation. Since the windows are made on every side of the chamber, it is possible to perform the fluorescence and transmission measurements at the same time. It is important to note that size of the system and field of view from the sample are adequate for X-ray spectrometer setup usage. This is especially important for the dispersive type spectrometers working at relatively short radius of curvature $(<0.5 \mathrm{~m})$ which requires space for detector operation in the immediate environment of the sample.

In case of measurements performed in different atmosphere than air, the side windows have to be sealed. Typical X-ray transparent windows are made of beryllium, graphite, quartz, or kapton. The design of the chamber allows for quick change of the windows and thus adaptation of material type and thickness according to experimental requirements. Other windows may be sealed by use of steel plugs and copper gaskets. The cell windows have no additional cooling system. Taking it into

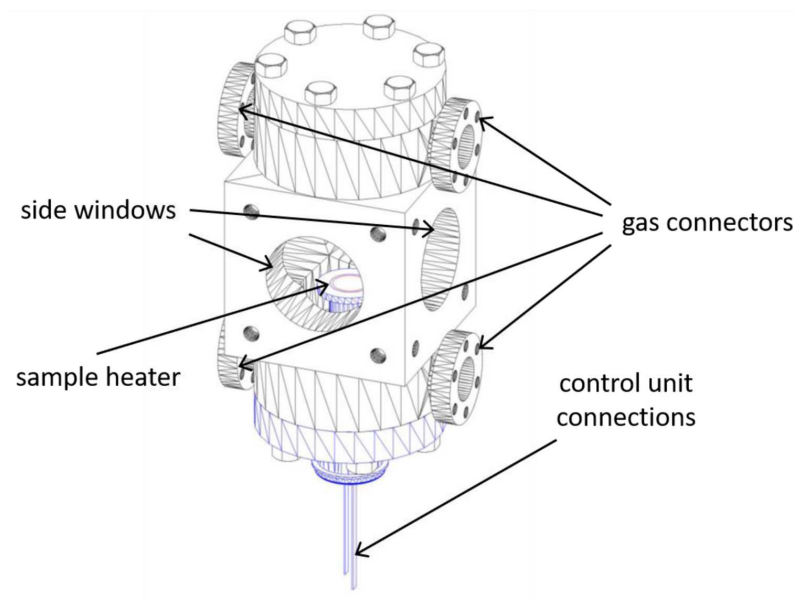

Fig. 1. The schematic design of the sample chamber. Four gas connectors allow for the continuous gas flow through chamber, without building the additional pressure inside. Side windows allow for performing measurements both in fluorescence and transmission mode. 


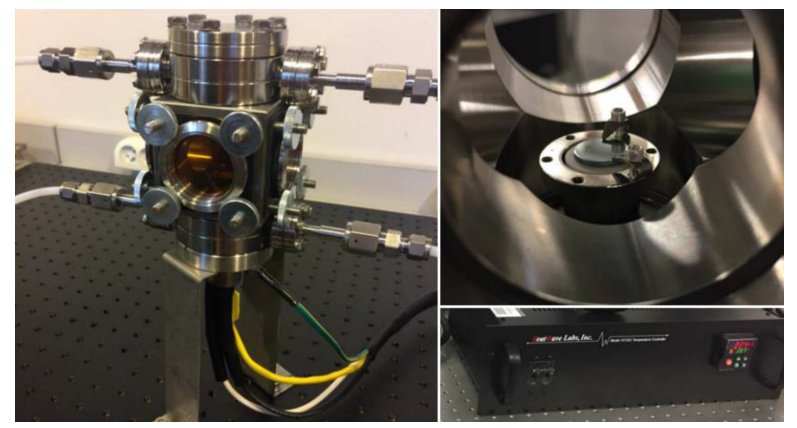

Fig. 2. Photographs of the setup for in situ thermal studies under different atmospheres: (left part) custommade cell with side windows sealed with a Kapton foil; (right top part) the inside of the sample chamber - the Ti metal disc is hold on a sample heater by a hook-like clips; (right bottom part) temperature controller unit.

consideration a test was performed to measure the external temperature of sample chamber, providing an information about maximum operation temperature. Temperature measurements were conducted both, with and without the kapton foil on windows. During the tests, the sample heater temperature was set to $500^{\circ} \mathrm{C}$ and maintained for $5 \mathrm{~h}$. Measurements revealed that in case of kapton foil installed, and windows sealed, the external temperature of sample chamber increased $c a$. $20^{\circ} \mathrm{C}$ above the ambient. In case of open windows, the external temperature grown only $10^{\circ} \mathrm{C}$ from the ambient. The maximum temperature in different atmosphere conditions is dependent on the heat resistance of the sealing material.

It is important to note that the cell has not been designed for high pressure measurements, but rather for comparable with ambient one with maximum difference of $10 \%$. To prevent pressure building inside the cell during measurements with different atmosphere than air, the additional gas connectors are mounted on both, top and bottom side of the cell. Owing to this, the setup allows for continues gas flow through the sample chamber, so the pressure inside is comparable to the atmospheric one.

Sample can be held on the heater unit by one to three screwed hook-like clips (see Fig. 2). The holder clips may vary in size which allows for better adjustment of sample with different size and thicknesses. The heater setup allows for holding circular shaped samples with maximum diameter of $12 \mathrm{~mm}$. The chamber setup may be attached to the base by use of four screwed cylindrical supporting legs. Since the supports are made of two parts, their length can be regulated. Adjusting lengths of the supports on one side allows to change the angle under which the radiation enters the chamber. The angle regulation is possible between 0 and $10^{\circ}$. For temperature control and setting, the sample heater, equipped with temperature sensor, is connected with the electronic controller unit (from HeatWave Labs, Inc.). The setup allows to program the heating profile with temperature and time accuracy of $1^{\circ} \mathrm{C}$ and $1 \mathrm{~s}$, respectively with the maximum allowed temperature of $1370^{\circ} \mathrm{C}$. The heating profile allows for setting the suitable heating rate, ranging from 1 to $50^{\circ} \mathrm{C} / \mathrm{min}$. The other possibility is to set the temperature to hold in required period of time, then change it and hold for another period. The heater setup gives also the possibility to gradually cool down the sample.

\subsection{Ex situ measurements}

In order to test the designed cell, the thermal oxidation of titanium discs was performed. The surface chemical composition of the samples heated at $300{ }^{\circ} \mathrm{C}$ and $500{ }^{\circ} \mathrm{C}$, was studied ex situ by means of X-ray photoelectron spectroscopy (XPS). The surface composition of the studied discs, determined based on the Ti $2 p$, O $1 s$, and $\mathrm{C} 1 s$ regions, is summarized in Table I. It should be kept in mind that carbon detected on the surface (at similar content in both samples) corresponds to carbon-based contamination. As can be seen, raising the temperature causes only a slight increase in $\mathrm{Ti}$ and $\mathrm{O}$ content on the surface.

In order to gain detailed information on chemical bonding of species present on the surface, the deconvolution of high-resolution spectra ( $\mathrm{Ti} 2 p$ and $\mathrm{O} 1 s$ ) was performed. The Ti $2 p$ spectra can be fitted with two peaks, representing the $\mathrm{Ti} 2 p_{3 / 2}$ and $\mathrm{Ti} 2 p_{1 / 2}$ states (shown in Fig. 3a). The respective peaks at $458.5 \pm 0.1 \mathrm{eV}$ and $464.2 \pm 0.1 \mathrm{eV}$ are attributed to the $\mathrm{Ti}^{4+}$ species in $\mathrm{TiO}_{2}$ [14]. It should be noted that no additional

TABLE I

Surface concentration of titanium, oxygen and carbon detected in titanium disc after thermal oxidation in $300^{\circ} \mathrm{C}$ and $500^{\circ} \mathrm{C}$ by XPS.

\begin{tabular}{c|c|c|c}
\hline \hline \multirow{2}{*}{ Sample } & \multicolumn{3}{|c}{ Surface concentration [at.\%] } \\
\cline { 2 - 4 } & Ti $2 p$ & O $1 s$ & C $1 s$ \\
\hline Ti_300C & 19.5 & 66.9 & 13.7 \\
Ti_500C & 19.8 & 67.4 & 12.8
\end{tabular}

(a)
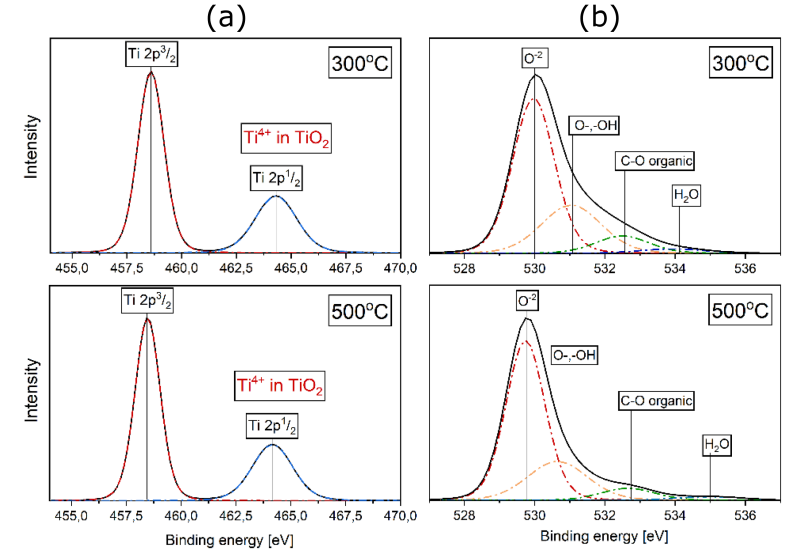

Fig. 3. Ti $2 p(\mathrm{a})$ and $\mathrm{O} 1 s$ (b) core-level spectra measured by XPS for $\mathrm{Ti}$ discs after thermal oxidation in $300^{\circ} \mathrm{C}$ and $500{ }^{\circ} \mathrm{C}$. 
peak can be observed in the Ti $2 p$ spectra, which confirms oxidation of metallic $\mathrm{Ti}$ to $\mathrm{TiO}_{2}$ in both studied temperatures. The $\mathrm{O} 1 s$ spectra were deconvoluted into four peaks (see Fig. 3b) [15, 16]. The prevailing peak with the lowest binding energy $(529.9 \pm 0.1 \mathrm{eV})$ can be assigned to lattice oxygen in metal oxides, while the second peak with higher binding energy $(530.9 \pm 0.2 \mathrm{eV})$ can be attributed to $\mathrm{O}^{-}$and hydroxyl species. The other peaks with low intensity, located at $532.6 \pm 0.1 \mathrm{eV}$ and $534.4 \pm 0.5 \mathrm{eV}$, correspond to $\mathrm{C}-\mathrm{O}$ contamination and water adsorbed on the surface, respectively.

\subsection{In situ measurements}

In this section, we demonstrate in situ XAS and XES measurements performed during the thermal oxidation of Ti disc, using the custom-made cell. For the purpose of the present study, we focus on the $\mathrm{Ti} K$-edge XAS and valence-to-core XES (v2c-XES) spectra. The combination of these techniques provides information about the electronic structure of occupied (valence band) and unoccupied (conduction band) states around the Fermi level [17]. The XAS and non-resonant XES spectra, collected at the beginning and end of the thermal oxidation process and reflecting the conduction and valence states of the material are plotted together in Fig. 4. It is important to note that no additional effects related to unexpected background signal or unwanted X-ray fluorescence from sample cell surroundings was observed. This means that the designed location and size of the windows as well as position of the sample/sample heater and other elements do not influence the spectral quality. The initial state, which corresponds to metallic Ti before thermal treatment, is characterized by a gapless valence and conduction electronic structure (see Fig. 4, top part). This is consistent to what is expected from a metallic phase. Along with rising temperature, the oxygen ligands are bound to metallic centre, which results in increase of oxidation state from $\mathrm{Ti}^{0}$ to $\mathrm{Ti}^{4+}$ in the formed $\mathrm{TiO}_{2}$.

This in turn causes blue shift of the raising absorption edge energy and appearance of pre-edge structures. The XAS pre-edge structure of $\mathrm{TiO}_{2}$ originates from a combination of dipole and quadrupole transitions. Namely, the $1 s \rightarrow d$ quadrupole transitions (low excitation probability) are characterized by a higher occurrence probability due to hybridization of the $d$ and $p$ states (more intense dipole-like excitation) [18, 19]. The final state, collected after oxidation at $700^{\circ} \mathrm{C}$, is characterized by the presence of two characteristic signals (see Fig. 4, bottom part). The first one is associated with the remaining metallic phase, while the second one is consistent with the formation of $\mathrm{TiO}_{2}$. In accordance with the literature [20, 21], the collected XAS spectra exhibit clearly visible and well-resolved pre-edge structure. The pre-edge features correspond to the transitions from the $1 s$ state to the localized and delocalized $3 d$ states of titanium, which are additionally partially hybridized with the $p$-type states. In the non-resonant XES spectra, weak structures are observed that are related to

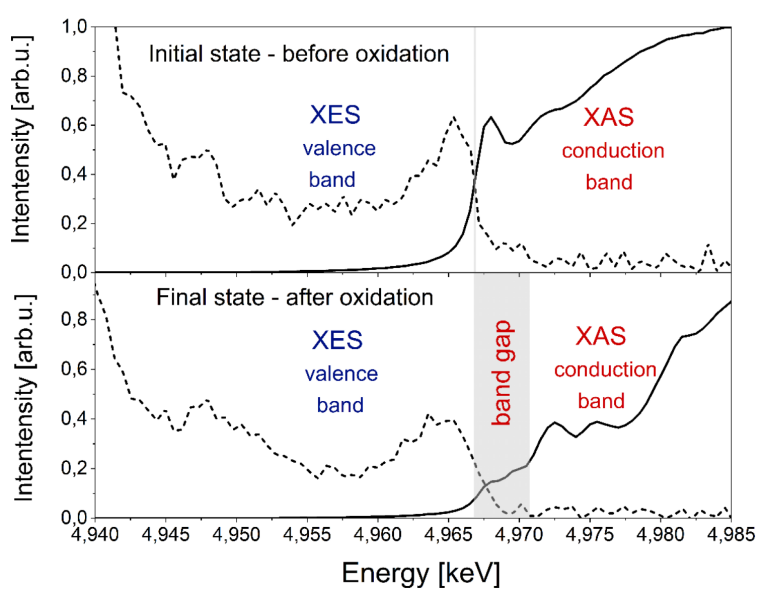

Fig. 4. Electronic band structure of titanium at different stages of thermal oxidation process determined from collected XAS and XES spectra. Top part: valence and conduction bands before oxidation showing a form of metallic titanium (without band gap). Bottom part: valence and conduction bands after oxidation in $700{ }^{\circ} \mathrm{C}$ showing a titanium dioxide as a major component (with marked approximate location of band gap).

the transition of electrons from the valence shells to the $1 s$ state. The thermal oxidation of $\mathrm{Ti}$ also affects the occupied electronic site, due to the appearance of additional oxygen states. The broadening of the structure located at an emission energy of $4965 \mathrm{eV}$ is attributed to the hybridization of $\mathrm{O} 2 p$ states with the occupied Ti $3 d$ states $[17,22]$. As shown, the measured valence and conduction band electronic states for $\mathrm{TiO}_{2}$ are separated by energy band gap, which is consistent with results expected for semiconductor. However, it should be mentioned that the XAS-XES measurements provide a band gap-like information, which is rather an averaged distance between $p$-projected occupied and unoccupied density of states. This is mainly because the estimated energy band gap value is affected by the core-hole screening effects $[17,21]$.

\section{Conclusions}

In this work, we have developed and tested a novel cell for ex situ and in situ thermal studies under different atmospheres with X-ray absorption and X-ray emission spectroscopy. The combination of experimental setup with hard X-rays from synchrotron sources gives a unique opportunity to map occupied and unoccupied electronic states of material under material working conditions.

\section{Acknowledgments}

The authors would like to thank Prof. J. Szlachetko from Institute of Nuclear Physics, Polish Academy of Sciences for comments, suggestions, and discussions. Authors would also like to acknowledge National Science Centre, Poland (NCN) for partial support under grant no. $2015 / 18 /$ E/ST3/00444. 


\section{References}

[1] R.B.P. Marcelino, C.C. Amorim, Environ. Sci. Pollut. Res. 26, 4155 (2019).

[2] B. Hammer, J.K. Nørskov, Adv. Catal. 45, 71 (2000).

[3] I. Pletikosić, M.N. Ali, A.V. Fedorov, R.J. Cava, T. Valla, Phys. Rev. Lett. 113, 216601 (2014).

[4] R. Köferstein, L. Jäger, S.G. Ebbinghaus, Solid State Ion. 249, 1 (2013).

[5] D. Gall, M. Städele, K. Järrendahl, I. Petrov, P. Desjardins, R.T. Haasch, T.Y. Lee, J.E. Greene, Phys. Rev. B 63, 125119 (2001).

[6] G. Agostini, D. Meira, M. Monte, et al., J. Synchrotr. Rad. 25, 1745 (2018).

[7] F.W. Lytle, P.S.P. Wei, R.B. Greegor, G.H. Via, J.H. Sinfelt, J. Chem. Phys. 70, 4849 (1979).

[8] F.W.H. Kampers, T.M.J. Maas, J. van Grondelle, P. Brinkgreve, D.C. Koningsberger, Rev. Sci. Instrum. 60, 2635 (1989).

[9] G.L. Chiarello, M. Nachtegaal, V. Marchionni, L. Quaroni, D. Ferri, Rev. Sci. Instrum. 85, 074102 (2014).

[10] R.C. Nelson, J.T. Miller, Catal. Sci. Technol. 2, 461 (2012).

[11] X-ray Absorption Fine Structure for Catalysts and Surfaces, Series on Synchrotron Radiation Techniques and Applications, Vol. 2, Ed. Y. Iwasawa, World Sci., River Edge (NJ) 1996.
[12] J. Sá, Y. Kayser, C.J. Milne, D.L. Abreu Fernandes, J. Szlachetko, Phys. Chem. Chem. Phys. 16, 7692 (2014).

[13] K. Wojtaszek, A. Wach, J. Czapla-Masztafiak, K. Tyrala, J. Sá, L. Yıldız Özer, C. Garlisi, G. Palmisano, J. Szlachetko, J. Synchrotr. Rad. 26, 145 (2019).

[14] L. Matějová, K. Kočí, M. Reli, et al., Appl. Catal. B 152, 172 (2014).

[15] G. Li, D. Zhang, J.C. Yu, Phys. Chem. Chem. Phys. 11, 3775 (2009).

[16] X. Gao, Y. Jiang, Y. Zhong, Z. Luo, K. Cen, J. Hazard. Mater. 174, 734 (2010).

[17] J. Szlachetko, J. Sá, Cryst. Eng. Comm. 15, 2583 (2013).

[18] F. de Groot, G. Vanko, P. Glatzel, J. Phys. Condens. Matter 21, 104207 (2009).

[19] P. Glatzel, M. Sikora, M. Fernández-García, Eur. Phys. J. Spec. Top. 169, 207 (2009).

[20] Z.Y. Wu, J. Zhang, K. Ibrahim, D.C. Xian, G. Li, Y. Tao, T.D. Hu, S. Bellucci, A. Marcelli, Q.H. Zhang, L. Gao, Z.Z. Chen, Appl. Phys. Lett. 80, 2973 (2002).

[21] J. Szlachetko, K. Michalow-Mauke, M. Nachtegaal, J. Sá, J. Chem. Sci. 126, 511 (2014).

[22] J. Sa, C. Garlisi, G. Palmisano, J. Czapla-Masztafiak, Y. Kayser, J. Szlachetko, Mater. Chem. Phys. 208, 281 (2018). 